\title{
Formation of Strontium Titanate Bicrystal by the Spark Plasma Sintering Method
}

\author{
L.A Hughes and K. van Benthem ${ }^{1}$ \\ ${ }^{1 .}$ Department of Chemical Engineering and Materials Science, University of California, Davis, CA USA
}

Spark plasma sintering (SPS) is a technique used for enhanced consolidation of powdered compacts by utilization of pulsed direct current and high uniaxial pressure. Physical mechanisms for densification of these powders as well as subsequent grain growth anomalies are debated in literature [1]. Limited information is available regarding the influence of electric fields on grain boundary formation and resulting core structures [2]. Bicrystals, created by manipulation of two single crystals to form a grain boundary with selected orientation, are the ideal method for study of fundamental grain boundary structures as a function of processing effects [3]. Forming bicrystals via parameters used in SPS provides an avenue to determine the impact of electric fields on grain boundary formation. In this study, strontium titanate $\left(\mathrm{SrTiO}_{3}\right)$ bicrystals were successfully formed, for the first time, at high pressure with moderate temperatures and times by SPS apparatus. Scanning electron microscopy (SEM) determined the quality of the interface bonding. Transmission electron microscopy (TEM) techniques combined with spatially resolved electron energy-loss spectroscopy (EELS) showed atomically resolved grain boundaries with no secondary phases.

Two single (100) $\mathrm{SrTiO}_{3}$ crystals were fixed with a nominal misorientation of $0^{\circ}, 4^{\circ}$, or $45^{\circ}$ around $<100>$ axis and then positioned within a SPS apparatus (Fig. 1). Bonding temperatures and times were systematically altered to maximize quality of the bonded interface. Uniaxial pressure of $120-140 \mathrm{MPa}$ was applied to minimize contact resistance. A 12-2 DC pulse sequence was used with a pulsed bias of $4 \mathrm{~V}$ and direct current of 550A. Fraction of interface bonding was characterized via SEM. Bicrystals with a $0^{\circ}$ twist orientation had a bonded interface greater than $95 \%$ at temperatures of $600^{\circ} \mathrm{C}$ and $700^{\circ} \mathrm{C}$ for a bonding time of 90 minutes. A $4^{\circ}$ twist angle bicrystal was formed at a temperature of $800^{\circ} \mathrm{C}$ for 20 minutes with a bonded interface of $70 \%$. Lastly, a $45^{\circ}$ twist angle bicrystal was formed at a temperature of $800^{\circ} \mathrm{C}$ for $90 \mathrm{~min}$ with a bonded interface of $45 \%$. Bonding parameters were altered for $4^{\circ}$ and $45^{\circ}$ twist orientation to increase interface bonding. High angle misorientation boundaries have a large structural mismatch between the two half-crystals, which leads to higher interfacial stress states during bicrystal formation and hinders cross-boundary diffusion. A focused ion beam instrument was used to prepare cross-sectional TEM samples for high-angle annular dark field (HAADF) imaging by scanning TEM (STEM). HAADF-STEM images of the $4^{\circ}$ twist bicrystal show an atomically ordered boundary with periodic distortions (Fig. 2). These distortions appear similar to screw dislocations found at the boundary of low angle twist $\mathrm{SrTiO}_{3}$ bicrystals by Fitting et al [4]. HAADF-STEM images of the $45^{\circ}$ twist bicrystal show an atomically ordered boundary with no secondary phases or amorphous films (Fig. 3). Spatially resolved EELS across the boundary coincides with EELS data from $\mathrm{SrTiO}_{3}$ bicrystals with twist boundaries formed by conventional methods [4]. Thus, the first successful formation of $\mathrm{SrTiO}_{3}$ bicrystals by SPS apparatus is reported. Future work will be focused on forming bicrystals by SPS technique to systematically study the effect of electric fields and heating rates on specific grain boundary structures. [4]

References:

[1] Z.A. Munir, U. Anselmi-Tamburini, M. Ohyanagi, Journal of Materials Science, 41 (2006) pp. 763. 
[2] S.C. Byeon, K.S. Hong, Materials Science and Engineering: A, 287 (2000) pp. 159.

[3] T. Gemming et al, Journal of the American Ceramic Society, 86 (2013) pp. 581.

[4] L. Fitting et al, Ultramicroscopy, 106 (2006) pp. 1053.

This work was supported by NSF grant \#1148897 and University of California Laboratory Fee award \#12-LR-238313. Work at the Molecular Foundry was supported by the Office of Science, Office of Basic Energy Sciences, of the U.S. Department of Energy under Contract No. DEAC02-05CH11231.

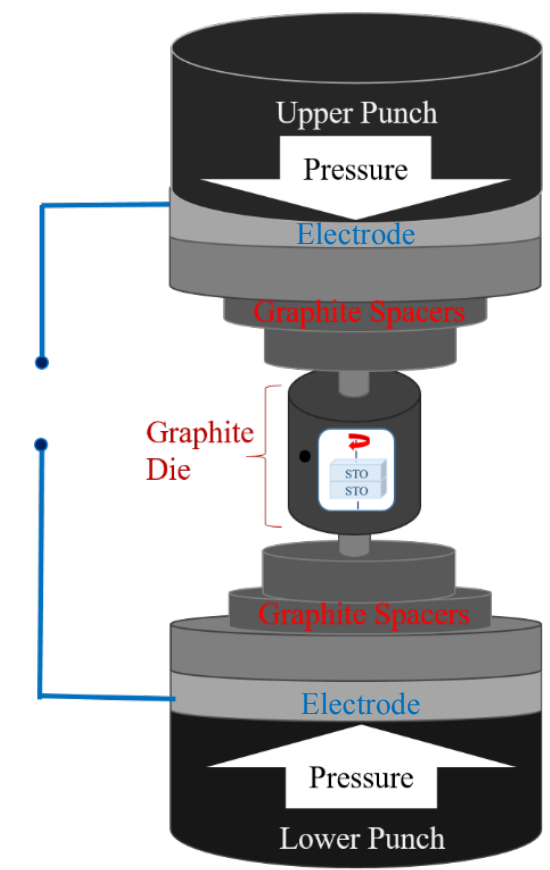

Figure 1: Schematic of experimental setup for $\mathrm{SrTiO}_{3}$ bicrystal formation by SPS apparatus

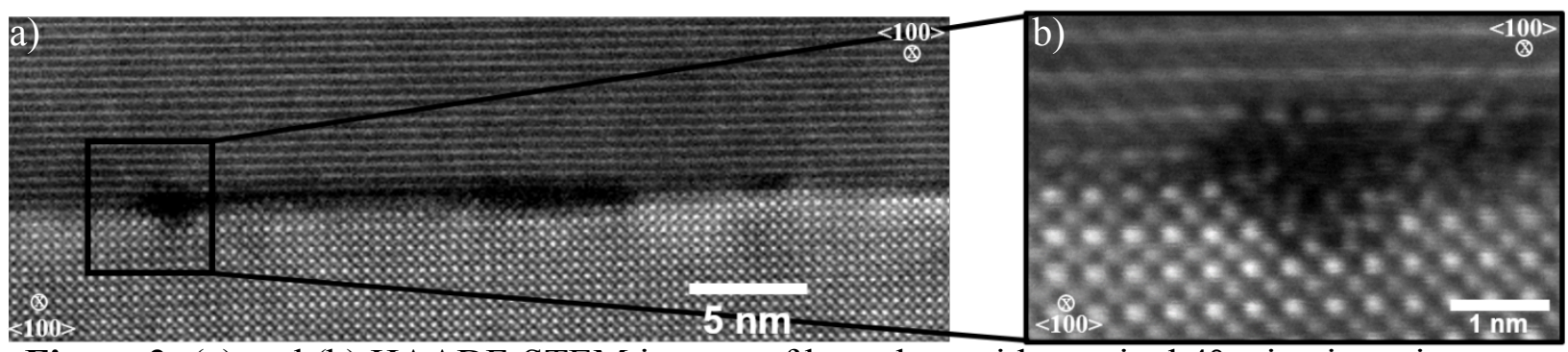

Figure 2: (a) and (b) HAADF-STEM images of boundary with nominal $4^{\circ}$ misorientation, recorded in $<100>$ zone axis. Bicrystal formed at $800^{\circ} \mathrm{C}$ for 20 minutes.

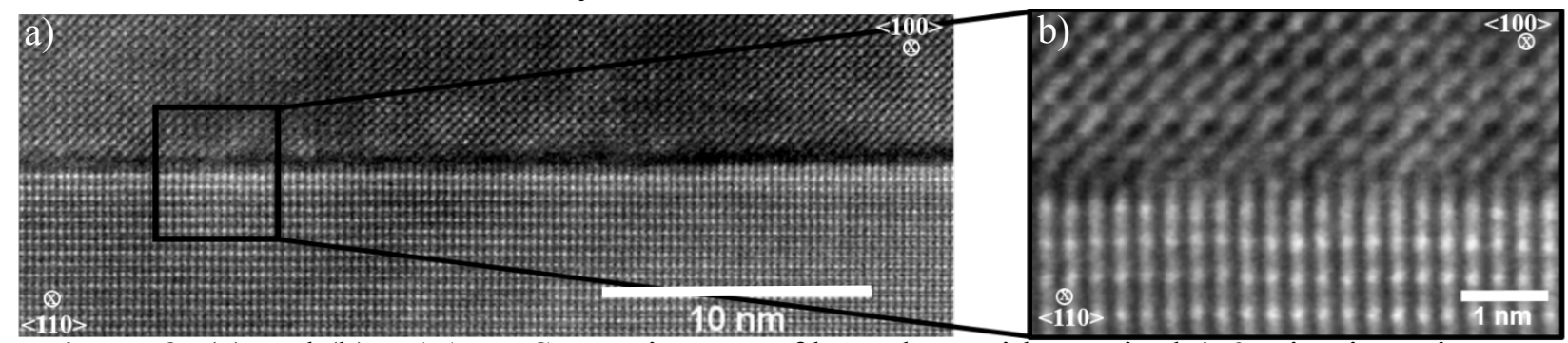

Figure 3: (a) and (b) HAADF-STEM images of boundary with nominal $45^{\circ}$ misorientation, recorded in $<110>$ zone axis. Bicrystal formed at $800^{\circ} \mathrm{C}$ for 90 minutes. 